\title{
Avaliação dos resultados obtidos nos grupos de controle do tabagismo realizados numa unidade de saúde de Curitiba-PR
}

\author{
Evaluation of the results obtained in the smoking control groups performed at a health unit \\ in Curitiba-PR
Evaluación de los resultados obtenidos en los grupos de control del tabaquismo realizados en una unidad de salud de Curitiba-PR

Lucileyne Wittkowski, Cynthia Ramos Soares Dias

\section{Resumo}

Introdução: O tabagismo é fator de risco para diversas doenças, sendo responsável por seis óbitos a cada segundo. No Brasil, sua prevalência é de 14,7\%. A terapia cognitivo comportamental é alicerce principal para esta abordagem. Objetivo: Avaliar os resultados obtidos nos grupos do programa nacional de controle do tabagismo (PNCT) desenvolvidos numa unidade municipal de saúde (UMS) de Curitiba - Paraná (PR), com ênfase na avaliação da taxa de cessação da dependência nicotínica e análise do perfil clínico epidemiológico dos pacientes. Métodos: Trata-se de um estudo descritivo, observacional e retrospectivo. Foram analisados dados da população participantes dos grupos do PNCT através das fichas da avaliação inicial e das planilhas de consolidação de informações do Ministério da Saúde. Resultados: Foram realizados 8 grupos, totalizando 160 tabagistas. $68 \%$ eram do sexo feminino, 38\% eram casados e $32,5 \%$ tinham ensino fundamental incompleto. A média de idade foi de 47,2 anos e de início do tabagismo, de 15 anos. A porcentagem de cessação do uso do tabaco variou de 12,5 até 72,7 ao final do primeiro mês. A taxa de desistência do tratamento chegou a 62,5\% nos grupos. Conclusão: Os Grupos do Programa de Controle ao Tabagismo apresentam resultados a curto prazo, porém alto índice de abandono do tratamento. Houve baixa adesão à manutenção da abstinêcia no decorrer do tempo. Maiores esforços devem ser destinados visando uma abstinência duradoura.

\begin{abstract}
Introduction: Smoking is a risk factor for several diseases and is responsible for six deaths every second. In Brazil, its prevalence is $14.7 \%$. Cognitive behavioral therapy (CBT) is the cornerstone for this approach. Objective: To evaluate the results obtained in the National Program of Tobacco Control (PNCT) groups developed at a Municipal Health Unit (UMS) in Curitiba - Paraná (PR), with emphasis on the evaluation of the cessation rate of nicotinic dependence and analysis of the epidemiological clinical profile of patients. Methods: It is a descriptive, observational and retrospective study. Data from the population participating in the PNCT groups were analyzed through the initial evaluation records and information consolidation spreadsheets of the Ministry of Health (MS). Results: Eight groups were performed totalizing 160 smokers. $68 \%$ were women, $38 \%$ were married and $32.5 \%$ had incomplete fundamental teaching. The average age was 47.2 years and the onset of smoking was 15 years. The percentage of cessation of tobacco use ranged from 12.5 to 72.7 at the end of the first month. The dropout rate reached $62.5 \%$ in the groups. Conclusion: The Tobacco Control Program Groups have results in the short term, but high index of abandonment of the treatment. There was low adherence to abstinence maintenance over time. Greater efforts should be directed towards lasting abstinence.
\end{abstract}

Como citar: Wittkowski L, Dias CRS. Avaliação dos resultados obtidos nos grupos de controle do tabagismo realizados numa unidade de saúde de Curitiba-PR. Rev Bras Med Fam Comunidade. 2017;12(39):1-11. http://dx.doi.org/10.5712/rbmfc12(39)1463

\section{Palavras-chave:}

Tabagismo

Uso de Tabaco

Abandono do Uso de Tabaco

Terapia Cognitiva

Keywords:

Tobacco use disorder

Tobacco Use

Tobacco Use Cessation

Cognitive Therapy
Fonte de financiamento: declaram não haver. Parecer CEP: não se aplica.

Conflito de interesses: declaram não haver. Procedência e revisão por pares: revisado por pares. Recebido em: 02/02/2017. Aprovado em: 07/02/2018. 


\section{Resumen}

Introducción: El tabaquismo es factor de riesgo para diversas enfermedades siendo responsable de seis muertes cada segundo. En Brasil, su prevalencia es del 14,7\%. La terapia cognitiva conductual (TCC) es la base principal para este abordaje. Objetivo: Evaluar los resultados obtenidos en los grupos del Programa Nacional de Control del Tabaquismo (PNCT) desarrollados en una Unidad Municipal de Salud (UMS) de Curitiba - Paraná (PR), con énfasis en la evaluación de la tasa de cesación de la dependencia nicotínica y análisis del perfil clínico epidemiológico de los pacientes. Métodos: Se trata de un estudio descriptivo, observacional y retrospectivo. Fueron analizados datos de la población participante de los grupos del PNCT por medio de las fichas de evaluación inicial y las plantillas de consolidación de informaciones del Ministerio de la Salud (MS). Resultados: Fueron realizados 8 grupos, totalizando 160 tabaquistas. $68 \%$ eran del sexo femenino, $38 \%$ estaban casados y $32,5 \%$ habían cursado la Educación Fundamental incompleta. El promedio de edad fue de 47,2 años y de inicio del tabaquismo de 15 años. El porcentaje de cesación del uso del tabaco ha variado de $12,5 \%$ a $72,7 \%$ al final del primer mes. La tasa de desistimiento del tratamiento llegó a los $62,5 \%$ en los grupos. Conclusión: Los Grupos del Programa de Control al Tabaquismo presentan resultados a corto plazo, pero alto índice de abandono del tratamiento. Hubo una baja adhesión al mantenimiento de la abstinencia en el transcurso del tiempo. Mayores esfuerzos deben ser destinados para una abstinencia duradera.
Palabras clave:

Tabaquismo

Uso del Tabaco

Cese del Uso de Tabaco

Terapia Cognitiva

\section{Introdução}

Estima-se que existam mais de 1 bilhão de fumantes no mundo, com cerca de 5,4 milhões de óbitos anualmente em nível internacional, sendo que o tabagismo encontra-se entre as principais causas de mortes evitáveis, devendo alcançar mais de 10 milhões de mortes anuais em 2030.1,2

De acordo com dados do Vigitel/2016, o percentual de adultos fumantes nas 27 capitais brasileiras foi de $10,2 \%$, sendo $12,7 \%$ para homens e $8 \%$ para mulheres. Dados da região Sul do país mostraram uma maior frequência de adultos fumantes em Curitiba (PR), com 17,8\% para o sexo masculino e 10,7 para feminino. ${ }^{3}$

Os fatores que se relacionam, tanto em nível nacional quanto internacional, ao hábito de fumar são: homens, negros e pardos, baixa escolaridade e população desfavorecida de centros urbanos. ${ }^{1}$ A prevalência é maior em pessoas etilistas ou que apresentem entes próximos dependentes da nicotina, sendo que o início do ato de fumar vem acontecendo nas faixas etárias cada vez mais baixas. ${ }^{1}$

Entre um terço e metade dos tabagistas morrem em média 15 anos mais precocemente. ${ }^{4}$ Além disso, o cigarro é responsável por 40 a $45 \%$ de todos os óbitos por neoplasias, ocorrendo em torno de 6 óbitos a cada segundo. ${ }^{1,5,6}$ Associado à alta mortalidade, o custo financeiro fornecido para atendimentos médicos, perda de produtividade e o próprio desfecho "morte" fazem do tabagismo um problema de saúde pública. ${ }^{1}$ Ademais, o tabagismo passivo é considerado a terceira maior causa de morte evitável, sendo responsável por cerca de 600 mil mortes anuais entre não fumantes. ${ }^{1,2}$

Tendo em vista que a vontade de parar de fumar está presente em $80 \%$ dos indivíduos que usam tabaco, sendo que destes apenas $3 \%$ realmente conseguem cessar a dependência a cada ano, o incentivo ao tratamento da dependência nicotínica foi colocado em pauta. ${ }^{5,7}$

Em meados de 2000, o Instituto Nacional do Câncer, por meio do Programa Nacional de Controle do Tabagismo (PNCT), desenvolveu o $1^{\circ}$ Encontro Nacional de Consenso sobre Abordagem e Tratamento do Fumante, no qual foram definidas as principais recomendações sobre a abordagem e manejo do paciente tabagista. Com foco na cessação do tabagismo, a Terapia Cognitivo-Comportamental (TCC) tornou-se alicerce principal desta abordagem, associada a apoio medicamentoso conforme condições específicas. ${ }^{7}$ 
Além disso, objetivou a implantação desta iniciativa em todas as Unidades de Saúde do país, expandindo a educação sobre o parar de fumar a todos os profissionais da área da saúde, capacitando-os a introduzir em sua prática cotidiana uma abordagem eficaz ao tabagismo. ${ }^{5}$

Porém, desde 1996, o Brasil iniciou o conceito de "fumódromo", trazendo em ascensão o controle do uso e divulgação do cigarro, por intermédio da Lei Federal no 9294/1996.8,9 Após 15 anos, a Lei Federal no 12546/2011 vetou o tabagismo em ambientes coletivos. ${ }^{9}$ Estudo que avaliou programas de promoção de saúde em 1600 Unidades de Saúde brasileiras demonstrou que 54\% possuem atividades de controle do tabagismo. ${ }^{10}$

O objetivo do presente estudo foi avaliar os resultados obtidos nos Grupos do PNCT desenvolvidos numa Unidade Municipal de Saúde de Curitiba (PR), durante o período de setembro de 2015 a setembro de 2016, associado à análise do perfil clínico epidemiológico dos pacientes participantes da estratégia.

\section{Métodos}

O desenho deste estudo caracteriza-se pelo delineamento Descritivo Observacional Retrospectivo.

Os grupos do PNCT são realizados sob autorização do Ministério da Saúde (MS) numa Unidade Municipal de Saúde de Curitiba (PR). Os grupos são baseados na TCC, com duração de 1 ano, sendo estruturados por meio de 4 sessões, com intervalos semanais no primeiro mês, durando de 1 a 2 horas, seguidos de 2 sessões com intervalos quinzenais no segundo mês, passando para encontros mensais de manutenção, realizados em grupo ou individual até completar 1 ano. A TCC é complementada por consultas médicas para avaliação da necessidade de apoio medicamentoso.

Anteriormente ao início do grupo, os participantes passam por preenchimento da Ficha de Avaliação Inicial constando dados pessoais, história tabágica e história clínica. Além disso, o MS exige controle rigoroso da evolução das sessões, dispensação dos manuais e das medicações com o preenchimento e envio trimestral de Planilhas de Consolidação de Informações contendo tais informações. ${ }^{11}$

O Programa de Controle do Tabagismo pode ser ministrado por qualquer profissional de saúde, desde que instruído para tal. Cursos de capacitação são oferecidos pela Prefeitura Municipal de forma obrigatória para o desenvolvimento da abordagem. Neste estudo, os grupos foram manejados por dois médicos residentes habilitados. Além disso, é necessário espaço físico disponível. A Unidade de Saúde onde foi desenvolvido a abordagem opera sob o modelo de Estratégia Saúde da Família (ESF), possuindo aproximadamente 20.000 habitantes adscritos vinculados a 4 Equipes de Saúde da Família (eSF).

A população da presente pesquisa foi composta pelos tabagistas que participaram dos grupos. Utilizaram-se os dados referentes às Fichas de Avaliação Inicial e as Planilhas de Consolidação de Informações do MS dos grupos desenvolvidos entre o período de setembro de 2015 a setembro de 2016. No final deste período, contato telefônico/consulta presencial foi realizado, pelos responsáveis dos grupos, a fim de verificar a manutenção da abstinência da amostra, através de questionamento direto sobre a permanência ou não da abstinência.

As variáveis analisadas relacionadas aos participantes foram as que constam na Ficha de Avaliação Inicial, incluindo dados sociais gerais de identificação, história do uso do tabaco, Teste de Fagerström, grau 
de motivação, história clínica - fatores de risco associados/contra indicações do apoio medicamentoso, comorbidades psiquiátricas, indicação de apoio medicamentoso e evoluções. Já as variáveis referentes aos grupos envolviam dispensação de manuais e medicações, presença nas sessões, apoio medicamentoso, cessação ou não do tabagismo.

Assim, foram incluídos na análise dos dados os pacientes tabagistas que passaram pelo preenchimento da Ficha de Avaliação Inicial e que participaram dos grupos com assiduidade nas sessões. Faltosos sem a devida justificativa e ausência/preenchimento incompleto da Ficha de Avaliação Inicial foram excluídos da amostra.

Os resultados foram coletados e digitalizados em planilhas do Microsoft Excel. Com o uso deste programa, a análise de dados foi realizada por meio de medidas de dispersão e medidas de tendência central.

A pesquisa foi submetida e aprovada pelo Comitê de Ética e Pesquisa da Secretaria Municipal da Saúde de Curitiba - PR, CAAE número 55403816.4.0000.0101, parecer número 1.552.735, aprovada no mês de maio de 2016.

\section{Resultados}

No período analisado, foram realizados 8 grupos de tabagismo, com um total de 160 pacientes, sendo que, destes, 40 foram convocados a participarem mais de uma vez, perfazendo uma amostra final de 120 novos pacientes.

Dos 120 tabagistas participantes, 82 (68\%) eram do sexo feminino, incluindo 6 (5\%) gestantes e 2 $(1,6 \%)$ em aleitamento materno. A Tabela 1 mostra a distribuição de gênero por grupo. A média de idade foi de 47,2 anos, variando de 19 a 74 anos.

Tabela 1. Distribuição do gênero por grupo - Unidade de Saúde Lotiguaçu-Curitiba/PR, 2016.

\begin{tabular}{cccc}
\hline Grupos & Feminino & Masculino & Total \\
\hline 1 & 25 & 7 & 32 \\
2 & 14 & 7 & 21 \\
3 & 16 & 4 & 20 \\
4 & 11 & 4 & 15 \\
5 & 13 & 8 & 21 \\
6 & 8 & 4 & 12 \\
7 & 15 & 7 & 22 \\
8 & 11 & 6 & 17 \\
Total & 113 & 47 & 160 \\
\hline
\end{tabular}

A Tabela 2 mostra o perfil de escolaridade, renda familiar e estado civil.

Em relação à idade de início do tabagismo, a média total foi de 15 anos, não havendo variação entre homens e mulheres. Dos 120 pacientes, 45 (37,5\%) referem medo de engordar após parar de fumar, sendo 35 (78\%) mulheres e 10 (22\%) homens. Apenas 18 (15\%) pacientes do total referem que o cigarro emagrece, sendo que, destes, 10 (55\%) relataram medo de aumento ponderal.

Ao analisar a história do uso do tabaco, obtiveram-se os resultados apresentados na Tabela 3. Dos $101(84 \%)$ pacientes que referem ter o cigarro como calmante, 90 (89\%) o utilizam durante a ansiedade. 
Tabela 2. Frequência de Escolaridade, Renda Familiar e Estado Civil - Unidade de Saúde Lotiguaçu-Curitiba/PR, 2016.

\begin{tabular}{|c|c|c|c|}
\hline & Variáveis & $n$ & $\%$ \\
\hline \multirow{9}{*}{ Escolaridade } & Sem escolaridade & 11 & 9,2 \\
\hline & Fundamental incompleto & 39 & 32,5 \\
\hline & Fundamental completo & 20 & 16,7 \\
\hline & Médio incompleto & 14 & 11,7 \\
\hline & Médio completo & 26 & 21,7 \\
\hline & Superior incompleto & 6 & 5,0 \\
\hline & Superior completo & 3 & 2,5 \\
\hline & Não sabe & 1 & 1,0 \\
\hline & Total & 120 & 100,0 \\
\hline \multirow{6}{*}{ Renda Familiar } & Até $2 \mathrm{SM}$ & 67 & 55,8 \\
\hline & De 2 até $4 \mathrm{SM}$ & 37 & 30,8 \\
\hline & De 4 até 7 SM & 4 & 3,3 \\
\hline & De 7 até 10 SM & 2 & 1,7 \\
\hline & Não sabe & 10 & 8,3 \\
\hline & Total & 120 & 100,0 \\
\hline \multirow{7}{*}{ Estado Civil } & Casado & 45 & 37,5 \\
\hline & Solteiro & 33 & 27,5 \\
\hline & Viúvo & 14 & 11,7 \\
\hline & Outro & 11 & 9,2 \\
\hline & Divorciado & 10 & 8,3 \\
\hline & Separado & 7 & 5,8 \\
\hline & Total & 120 & 100,0 \\
\hline
\end{tabular}

SM: salários mínimos.

Foi constatado que 20 (17\%) pacientes, da amostra total, residem sozinhos. Dos 100 (83\%) participantes restantes, aproximadamente 53 (50\%) pessoas convivem com fumante em casa.

Em relação a tentativas anteriores de abandonar tabagismo, 97 (81\%) participantes já tentaram cessar tabagismo em algum momento. Ao avaliar o uso de recursos para auxiliar na cessação ao tabagismo, evidencia-se que 94 (78\%) não utilizaram nenhuma ferramenta para ajudar no processo de cessação.

Sobre o Teste de Fagerström aplicado, foi observado que 28 (23\%) pacientes obtiveram pontuação de 6 no escore, 19 (16\%) pessoas com escore de 5, 17 (14\%) com nota 7 e 16 (13\%) com escore 8, os demais participantes ficaram amplamente divididos nos demais escores do teste.

No transcorrer dos grupos, obteve-se um total de convocados, equivalendo ao número total de pessoas convidadas a participarem. Destas, uma parcela não iniciou o tratamento, enquanto que outra parte foi desistindo do acompanhamento, perfazendo uma taxa de abandono. Dos que se mantiveram até o final da abordagem, foi contabilizado quantos conseguiram parar de fumar, produzindo a taxa de cessação ao tabagismo.

A Tabela 4 traz o perfil de evolução de cada um dos 8 grupos. Verifica-se a quantidade de convocados por grupo, quantos compareceram na primeira e quarta sessão (gerando a taxa de abandono por grupo desistência do tratamento). Quantifica-se, ainda, a cessação do tabagismo na última sessão e uso de medicação de apoio. Foram 160 pessoas convocadas a participar dos grupos, 85 (53\%) estiveram presentes no primeiro encontro e $50(31 \%)$ mantiveram frequência ao final do primeiro mês. A porcentagem de 
Tabela 3. História do uso do tabaco: razões para fumar, situações relacionadas ao tabaco e motivos para deixar de fumar.

\begin{tabular}{|c|c|c|c|}
\hline & Variáveis* & Sim & $\%$ \\
\hline \multirow{6}{*}{ Razões para fumar } & É um grande prazer & 51 & $43 \%$ \\
\hline & É muito saboroso & 35 & $29 \%$ \\
\hline & O cigarro acalma & 101 & $84 \%$ \\
\hline & É charmoso & 7 & $6 \%$ \\
\hline & Emagrece & 18 & $15 \%$ \\
\hline & Para distração & 43 & $36 \%$ \\
\hline \multirow{9}{*}{ Situações relacionadas ao tabaco } & Café & 79 & $66 \%$ \\
\hline & Após refeições & 95 & $79 \%$ \\
\hline & Bebida alcoólica & 46 & $38 \%$ \\
\hline & Alegria & 43 & $36 \%$ \\
\hline & Tristeza & 87 & $73 \%$ \\
\hline & Ansiedade & 102 & $85 \%$ \\
\hline & Telefone & 33 & $28 \%$ \\
\hline & Trabalho & 41 & $34 \%$ \\
\hline & Outra situação & 21 & $18 \%$ \\
\hline \multirow{11}{*}{ Motivos para deixar de fumar } & Sente que o tabaco está afetando a saúde & 97 & $81 \%$ \\
\hline & Pelo bem-estar da família & 77 & $64 \%$ \\
\hline & Familiares pedem, exceto filhos & 47 & $39 \%$ \\
\hline & Filhos pedem & 61 & $51 \%$ \\
\hline & Sente pressionado pelas pessoas & 27 & $23 \%$ \\
\hline & Fumar é antissocial & 52 & $43 \%$ \\
\hline & Gasto dinheiro com cigarro & 72 & $60 \%$ \\
\hline & Preocupa com saúde no futuro & 90 & $75 \%$ \\
\hline & Não quer ser dependente & 67 & $56 \%$ \\
\hline & Fumar é mal exemplo para crianças & 78 & $65 \%$ \\
\hline & Outro & 6 & $5 \%$ \\
\hline
\end{tabular}

*Variáveis independentes. Unidade de Saúde Lotiguaçu-Curitiba/PR, 2016.

abstinência ao cigarro no final do primeiro mês variou de 12,5 (grupo 3) até 72,7 (grupo 2). Analisando o percentual de abandono do grupo, o menor foi de 27,3\% (grupo 2) e o maior de 62,5\% (grupo 3). Em relação ao uso de medicação, a variação ocorreu de 25\% (grupo 3) para 100\% (grupo 6).

Tabela 4. Perfil de evolução de cada grupo - Unidade de Saúde Lotiguaçu-Curitiba/PR, 2016.

\begin{tabular}{|c|c|c|c|c|c|c|c|}
\hline Grupo & $\begin{array}{l}\text { Convocados } \\
\text { para grupo }\end{array}$ & $\begin{array}{l}\text { Participaram } \\
\text { da } 1^{\text {a }} \text { sessão }\end{array}$ & $\begin{array}{l}\text { Participaram } \\
\text { da } 4^{\mathrm{a}} \text { sessão }\end{array}$ & $\begin{array}{c}\text { Sem fumar } \\
\text { na } 4^{\text {a }} \text { sessão }\end{array}$ & $\begin{array}{c}\text { Abandono } \\
\text { (\%) }\end{array}$ & $\begin{array}{l}\text { Cessação } \\
(\%)\end{array}$ & $\begin{array}{c}\text { Usaram medicação } \\
(\%)\end{array}$ \\
\hline 1 & 32 & 21 & 11 & 10 & 47,6 & 47,6 & 66,7 \\
\hline 3 & 20 & 8 & 3 & 1 & 62,5 & 12,5 & 25,0 \\
\hline 4 & 15 & 13 & 9 & 9 & 30,8 & 69,2 & 69,2 \\
\hline 7 & 22 & 10 & 6 & 6 & 40,0 & 60,0 & 70,0 \\
\hline 8 & 17 & 8 & 5 & 5 & 37,5 & 62,5 & 37,5 \\
\hline Total & 160 & 85 & 50 & 45 & $x$ & $x$ & $x$ \\
\hline
\end{tabular}


Em relação à indicação de medicação, 54 (34\%) participantes usaram algum método disponível, distribuídos da seguinte forma: aproximadamente 43 (80\%) usaram somente adesivo, 8 (15\%) adesivo + bupropiona, 2 (4\%) somente a bupropiona e 1 (1\%) outras medicações.

Um dos conceitos bem trabalhados durante os grupos é a forma como o paciente escolhe para cessar tabagismo. Há três opções: abrupta (escolhido um dia para parar - "dia D”), gradual por redução (reduz a quantidade de cigarros fumados ao dia até cessar, num período não superior a sete dias) e gradual por adiamento (adia-se o horário para acender o primeiro cigarro do dia até cessar, num período não superior a sete dias). ${ }^{11} \mathrm{O}$ método de parada predominante escolhido pelos pacientes em todos os grupos foi o abrupto, apenas uma pessoa escolheu gradual por redução e outra gradual por adiamento.

Ao averiguar a manutenção da abstinência ao cigarro dos pacientes que findaram os grupos sem fumar, verificaram-se os resultados mostrados na Tabela 5. Convém ressaltar que após as quatro primeiras sessões, equivalente ao primeiro mês da realização dos grupos, todos os pacientes que se mantiveram sem tabaco foram convocados para as sessões de manutenção. Esta fase de acompanhamento foi organizada a cada 15 dias, no segundo mês, e mensal até completar um ano.

Tabela 5. Manutenção da abstinência no decorrer o tempo - Unidade de Saúde Lotiguaçu-Curitiba/PR, 2016.

\begin{tabular}{cccc}
\hline Grupo & Abstinentes $4^{\text {a }}$ sessão & Após acompanhamento & $\%$ \\
\hline 1 & 10 & 3 & 30,0 \\
2 & 8 & 4 & 50,0 \\
3 & 1 & 1 & 100,0 \\
4 & 9 & 2 & 22,2 \\
5 & 2 & 2 & 100,0 \\
6 & 4 & 1 & 25,0 \\
7 & 6 & 6 & 100,0 \\
8 & 5 & $X$ & $X$ \\
Total & 45 & 19 & $X$ \\
\hline
\end{tabular}

No presente estudo, a partir do terceiro mês não houve participação dos abstinentes. Dessa forma, no final do período analisado, foi verificado o status atual após cessação por meio de contato telefônico/ consulta presencial. Apenas os pacientes que se apresentaram sem fumar na quarta sessão dos grupos foram consultados.

A Tabela 5 deve ser interpretada com devido cuidado. Todos os grupos foram acompanhados no decorrer do tempo, porém o intervalo de tempo de seguimento de cada grupo é diferente. A presente pesquisa foi realizada no período de um ano (setembro/2015 a setembro/2016). A análise da manutenção da abstinência foi feita em setembro/2016, conforme explicado anteriormente. Assim, os dados referentes ao grupo 1 , realizado em setembro/2015, mostram acompanhamento completo após um ano. No período de março/2016 até setembro/2016 foi iniciado um grupo a cada mês. Sendo assim, o período de acompanhamento dos grupos descritos é: grupo 2 - 6 meses, grupo 3 - 5 meses, grupo 4 - 4 meses, grupo 5 - 3 meses, grupo 6-2 meses, grupo 7 - 1 mês, grupo 8 - não teve período de acompanhamento. 


\section{Discussão}

O perfil socioeconômico da amostra estudada nesta pesquisa caracterizou-se por uma população adulta, casada, com predomínio do sexo feminino e ensino fundamental incompleto, com renda familiar de até 2 salários mínimos. Dados semelhantes foram encontrados em estudo desenvolvido pelo HC/UNICAMP em que, dos 171 tabagistas avaliados, $73,4 \%$ eram mulheres, $48 \%$ casadas, $74,6 \%$ tinham escolaridade fundamental e $57 \%$ estavam trabalhando. ${ }^{12}$

A prevalência de tabagistas do sexo feminino, em análise feita nas capitais brasileiras em 2010, é de $12,4 \% .{ }^{13}$ Bittencourt et al., ${ }^{14}$ em seu estudo, detectaram que mulheres com ensino fundamental/médio apresentaram mais chances de pensar em cessar tabagismo comparadas com ensino superior após se depararem com advertências nos maços de cigarro.

Cessado o uso do tabaco, o paciente pode ter aumento ponderal, em torno de $5 \mathrm{~kg}$ no primeiro ano. ${ }^{1} \mathrm{Na}$ análise dos dados constatou-se que o medo de engordar existe, sendo o cigarro utilizado como calmante nos momentos de ansiedade. O pensamento de que o tabaco atua como calmante é equivocada. A nicotina atua na área de recompensa cerebral, liberando neurotransmissores. Entre eles, destaca-se a dopamina, que gera sensação curta de bem-estar, bom humor e hiperfoco, a norepinefrina e a acetilcolina que, juntas, provocam excitação, tornando o cigarro um estimulante. ${ }^{6}$

A cessação do tabagismo é uma tarefa difícil devido à dependência química, exigindo várias tentativas para se obter sucesso. Azevedo et al. ${ }^{12}$ mostraram que, de 171 tabagistas, $76 \%$ já tinham tentado parar de fumar, resultado próximo ao encontrado na atual pesquisa. Bittencourt et al. ${ }^{14}$ evidenciaram que pessoas do sexo feminino que tiveram vontade de parar no último ano apresentaram maior chance de abandonar tabaco comparadas às que nunca tentaram.

Houve grande variação, entre os grupos, tanto nas taxas de abandono do tratamento quanto nas taxas de cessação do tabagismo, porém 5 grupos tiveram taxas superiores a $50 \%$ de cessação do ato de fumar. Em grupo realizado numa Unidade de Saúde de Jundiaí, 12 fumantes participaram das sessões. Foram desenvolvidos 6 encontros com frequência semanal, obtendo uma taxa de $60 \%$ de cessação no final dos encontros. ${ }^{15}$

Correlacionando-se aos resultados obtidos, estudo desenvolvido num hospital público de Brasília constatou que dos 109 tabagistas participantes das 4 sessões de TCC semanal associado a apoio medicamentoso, 83,6\% cessaram tabagismo no final das sessões. Foi realizada avaliação de 3 grupos diferentes e detectada taxa de $43,8 \%$ de abstinência após 4 meses e 36,6\% após 6 meses de acompanhamentos. ${ }^{16} \mathrm{Em}$ contrapartida, Azevedo et al. ${ }^{12}$ encontraram percentual de 79,1 que pararam de fumar durante o tratamento. Avaliados após 25 meses, 62\% continuavam sem fumar.

Os dados fornecidos no presente estudo mostram altas porcentagens de desistência do tratamento. Ademais, o percentual que se manteve em abstinência após passar do tempo não é muito satisfatório. Estudo numa unidade de Saúde de Cuiabá/MT seguiu por um semestre 216 fumantes, obtendo $142(65,74 \%)$ pessoas que completaram tratamento em 6 meses, sendo que $74(34,26 \%)$ abandonaram o controle. ${ }^{17}$

Em uma revisão da Cochrane, autores concluíram que intervenções que combinem TCC com apoio medicamentoso aumentam o sucesso da cessação ao tabagismo comparado a intervenção mínima ou 
cuidados diários. ${ }^{18}$ Outra revisão sobre Grupos de Terapia Comportamental nos Programas Anti-Tabaco revela que terapia em grupo é melhor para ajudar as pessoas a parar de fumar do que a autoajuda e outras terapias menos intensivas, porém não há evidências de que a terapia em grupo seja mais eficaz que o aconselhamento individual intensivo. ${ }^{19}$

Há forte evidência de que algumas intervenções voltadas para o indivíduo aumentem as taxas de abandono do cigarro. Conselhos em grupo ou individual, terapia farmacológica e intervenções múltiplas são opções, obtendo o mesmo efeito quando realizados no ambiente de trabalho ou fora dele. Intervenções de autoajuda e suporte social foram menos efetivas. ${ }^{20}$

Associado à TCC, foi oferecido apoio medicamentoso, quando indicado, destacando-se o maior uso da terapia de reposição nicotínica (TRN) com adesivo. De acordo com revisão publicada pela Cochrane, qualquer tipo de TRN auxilia na manutenção da cessação do tabagismo. A TRN aumenta a taxa de abandono de 50 a 70\%, independentemente da forma. Comparando TRN à terapia não nicotínica (bupropiona), não há evidência na diferença de eficácia entre ambos. ${ }^{21}$ Da mesma forma, metanálise mostrou que tanto TRN quanto bupropiona são superiores ao placebo e ambos apresentam igual eficácia. ${ }^{22}$ Em relação ao método de parada, revisão evidenciou que não há taxas superiores ou inferiores de abstinência quando se compara método de parada gradual da abrupta. ${ }^{23}$

Lombardi et al., ${ }^{13}$ em seu estudo, revelam que o combate ao tabagismo deve ser entendido como papel de todos os profissionais da saúde. Estudo realizado em 7 cidades do Paraná, com objetivo de verificar os papéis dos trabalhadores comunitários de saúde nos programas de cessação do tabagismo, revelou que houve grande reconhecimento de que todos os setores administrativos desempenham papel importante no Programa de Controle do Tabagismo, menos na intervenção breve. Porém, apenas 48,4\% dos entrevistados afirmaram já terem participado de alguma forma no controle do tabagismo. ${ }^{24}$

Sobre a capacitação dos profissionais que desenvolvem os grupos, pesquisa publicada em 2014 revela que todos os profissionais devem passar pela capacitação antes de iniciar suas atividades no Programa. A maioria $(81,2 \%)$ dos profissionais de saúde indicaram que a capacitação é excelente ou boa, sendo suficiente para a prática. ${ }^{25}$

\section{Considerações finais}

Ao término deste trabalho, percebeu-se que Grupos do Programa de Controle do Tabagismo devem ser desenvolvidos devido ao grande impacto que o cigarro proporciona na saúde populacional. Ficou evidente que os grupos organizados pela Unidade de Saúde do presente estudo apresentam bons resultados, semelhantes ao que a literatura mostra.

Houve alto índice de abandono do tratamento e baixa adesão à manutenção da abstinêcia no decorrer do tempo. Maiores esforços devem ser destinados visando uma abstinência em longo prazo. A identificação do motivo para altas taxas de abandono dos grupos, o porquê da ausência de pacientes nas sessões de manutenção e o que leva os tabagistas a recaírem pode ser uma estratégia para compreender melhor o processo da permanência sem tabaco, podendo, dessa forma, ajudar a evitar o retorno da dependência nicotínica. 


\section{Referências}

1. Santos JDP, Achutti A, Guths P. Tabagismo. In: Duncan BB, Schmidt MI, Giugliani ERJ, eds. Medicina ambulatorial: condutas de atenção primária baseada em evidências. $4^{\mathrm{a}}$ ed. Porto Alegre: Artmed; 2013. p. 564-73.

2. Studart FS, Zavattieri AG. Tabagismo. In: Gusso G, Lopes JMC, eds. Tratado de Medicina de Família e Comunidade - Princípio, Formação e Prática. Porto Alegre: Artmed; 2012. p. 1943-80.

3. Brasil. Ministério da Saúde. Agência Nacional de Saúde Suplementar. Vigitel Brasil 2016: Saúde Suplementar. Vigilância de fatores de risco eproteção para doenças crôncias por inquértio telefônico. Estimativas sobre frequência e distribuição sociodemográfica de fatores de risco e proteção para doenças crônicas nas capitais dos 26 estados brasileiros e no Distrito Federal em 2016. Brasília: Ministério da Saúde; 2017.

4. World Health Organization. Reducing deaths from tobacco. In: World Health Statistics 2008. Geneva: WHO Library Cataloguing-inPublication Data; 2008. p. 18-20.

5. Brasil. Ministério da Saúde. Instituto Nacional de Câncer. Coordenação de Prevenção e Vigilância. Abordagem e Tratamento do Fumante - Consenso 2001. Rio de Janeiro: INCA; 2001.

6. Silva AR, Chiovatto ED, Sampaio M, Rachid L. Tabagismo. In: Medeiros Junior ME, Silva ATC, Alonso Junior AH, Lopes HR, Rios IC, Arrojo Junior JC, et al., orgs. Manual do Médico de Família Santa Marcelina. São Paulo: Martinari; 2016. p. 671-83.

7. Brasil. Ministério da Saúde. Instituto Nacional do Câncer. Tratamento do Tabagismo [Internet]. [acesso 2016 Mar 22]. Disponível em: http://www1.inca.gov.br/situacao/arquivos/acoes_tratamento_tabagismo.pdf

8. Madewell ZJ, Figueiredo VC, Harbertson J, Pérez RL, Novotny T. Exposure to smoking in soap operas and movies: smoking cessation and attempts to quit. Cad Saúde Pública. 2017;33(Suppl 3):e00118015. DOI: http://dx.doi.org/10.1590/0102-311x00118015

9. Mendes FL, Szklo AS, Perez CA, Cavalcante TM, Fong GT. A percepção do cumprimento das leis antifumo em bares e restaurantes em três cidades brasileiras: dados do ITC-Brasil. Cad. Saúde Pública. 2017;33(Suppl 3):e00140315. DOI: http://dx.doi.org/10.1590/0102$311 \times 00140315$

10. Ramos LR, Malta DC, Gomes GAO, Bracco MM, Florindo AA, Mielke GI, et al. Prevalência de programas de promoção da saúde em unidades básicas no Brasil. Rev Saúde Pública. 2014;48(5):837-44. DOI: http://dx.doi.org/10.1590/S0034-8910.2014048005249

11. Brasil. Ministério da Saúde. Coordenação de Prevenção e Vigilância — Conprev. Deixando de Fumar sem Mistérios. Rio de Janeiro: MS/ INCA; $2004.54 \mathrm{p}$.

12. Azevedo RCS, Higa CMH, Assumpção ISMA, Frazatto CRG, Fernandes RF, GoulartW, et al. Grupo terapêutico para tabagistas: resultados após seguimento de dois anos. Rev Assoc Med Bras. 2009;55(5):593-6. DOI: http://dx.doi.org/10.1590/S0104-42302009000500025

13. Lombardi EMS, Prado GF, Santos UP, Fernandes FLA. O tabagismo e a mulher: riscos, impactos e desafios. J Bras Pneumol. 2011;37(1):118-28. DOI: http://dx.doi.org/10.1590/S1806-37132011000100017

14. Bittencourt L, Person SD, Cruz RC, Scarinci IC. Pictorial health warnings on cigarette packs and the impact on women. Rev Saúde Pública. 2013;47(6):1123-9. DOI: http://dx.doi.org/10.1590/S0034-89102013000901123

15. Sousa NA. Abordagem e tratamento do tabagismo: resultados de um grupo educativo. In: I Fórum da Saúde: o foco é você. 2011 Out 7-9; Jundiaí, SP, Brasil.

16. Mesquita AA. Avaliação de um programa de tratamento do tabagismo. Rev Bras Ter Comp Cogn. 2013;15(2):35-44.

17. Pawlina MM, Rondina RC, Espinosa MM, Botelho C. Abandonment of nicotine dependence treatment: A cohort study. Sao Paulo Med J. 2016;134(1):47-55. DOI: http://dx.doi.org/10.1590/1516-3180.2015.00830309

18. Stead LF, Koilpillai P, Fanshawe TR, Lancaster T. Combined pharmacotherapy and behavioural interventions for smoking cessation. Cochrane Database Syst Rev. 2016;3:CD008286. DOI: http://dx.doi.org/10.1002/14651858.CD008286.pub3

19. Stead LF, Carroll AJ, Lancaster T. Group behaviour therapy programmes for smoking cessation. Cochrane Database Syst Rev. 2017;3:CD001007.

20. Cahill K, Lancaster T. Workplace interventions for smoking cessation. Cochrane Database Syst Rev. 2014;(2):CD003440.

21. Stead LF, Perera R, Bullen C, Mant D, Hartmann-Boyce J, Cahill K, et al. Nicotine replacement therapy for smoking cessation. Cochrane Database Syst Rev. 2012;11:CD000146. DOI: http://dx.doi.org/10.1002/14651858.CD000146.pub4 
22. Cahill K, Stevens S, Perera R, LancasterT. Pharmacological interventions for smoking cessation: an overview and network meta-analysis. Cochrane Database Syst Rev. 2013;(5):CD009329. DOI: http://dx.doi.org/10.1002/14651858.CD009329.pub2

23. Lindson-Hawley N, Aveyard P, Hughes JR. Reduction versus abrupt cessation in smokers who want to quit. Cochrane Database Syst Rev. 2012;11:CD008033. DOI: http://dx.doi.org/10.1002/14651858.CD008033.pub3

24. Bittencourt L, Scarinci IC. Is There a Role for Community Health Workers in Tobacco Cessation Programs? Perceptions of Administrators and Health Care Professionals. Nicotine Tob Res. 2014;16(5):626-31. DOI: http://dx.doi.org/10.1093/ntr/ntt217

25. Bittencourt L, Cruz RC, Scarinci IC. Seleção e capacitação para o tratamento do tabagismo no sistema único de saúde: perspectivas de gestores e profissionais de saúde no estado do Paraná, Brasil. Epidemiol Serv Saúde. 2014;23(4):645-54. DOI: http://dx.doi.org/10.5123/ S1679-49742014000400006

Prefeitura Municipal de Curitiba. Curitiba, PR, Brasil. lucy.witt@yahoo.com.br (Autora correspondente); cynmedi@ hotmail.com 